Available online on 25.06.2014 at http://iddtonline.info
Journal of Drug Delivery and Therapeutics
Open access to Pharmaceutical and Medical research
(C) 2014, publisher and licensee JDDT, This is an Open Access article which permits unrestricted noncommercial use, provided the original
work is properly cited

\title{
LIPOSOMES: AN OVERVIEW
}

\author{
Prabhakar Vishvakrama*, Saurabh Sharma \\ Pharmaceutics Research Lab, Department of Pharmaceutics, Vivek College of Technical Education, Bijnor, India
}

Email: prabhakar.vishvakarma7788@gmail.com

\begin{abstract}
The discovery of liposome or lipid vesicle emerged from self forming enclosed lipid bi-layer upon hydration. Liposome drug delivery systems have played a significant role in formulation of potent drug to improve therapeutics. Recently the liposome formulations are targeted to reduce toxicity and increase accumulation at the target site. There are several new methods of liposome preparation based on lipid drug interaction and liposome disposition mechanism including the inhibition of rapid clearance of liposome by controlling particle size, charge and surface hydration. Most clinical applications of liposomal drug delivery are targeting to tissue with or without expression of target recognition molecules on lipid membrane. The liposomes are characterized with respect to physical, chemical and biological parameters. The sizing of liposome is also critical parameter which helps characterize the liposome which is usually performed by sequential extrusion at relatively low pressure through polycarbonate membrane (PCM). This mode of drug delivery lends more safety and efficacy to administration of several classes of drugs like antiviral, antifungal, antimicrobial, vaccines, anti tubercular drugs and gene therapeutics. Present applications of the liposomes are in the immunology, dermatology, vaccine adjuvant, eye disorders, brain targeting, infective disease and in tumour therapy. The new developments in this field are the specific binding properties of a drug-carrying liposome to a target cell such as a tumor cell and specific molecules in the body (antibodies, proteins, peptides etc.); stealth liposomes which are especially being used as carriers for hydrophilic (water soluble) anticancer drugs like doxorubicin, mitoxantrone; and bisphosphonate- liposome mediated depletion of macrophages. This review would be a help to the researchers working in the area of liposomal drug delivery.
\end{abstract}

Keywords: Liposomes, Brain Targeting, Tumor Therapy

\section{INTRODUCTION}

Liposomes were discovered in the early 1960's by Bangham and collegue. The discovery of liposome or lipid vesicle emerged from self forming enclosed lipid bi-layer upon hydration. Liposomes, the phospholipid vesicles, are spherical lipid bilayers capable of entrapping water soluble solutes within an aqueous domains or alternatively lipid molecules within the lipid bilayers. They are biodegradable, biocompatible and non-immunogenic in nature, which makes them ideal drug carrier systems in therapeutics ${ }^{1}$.When phospholipids are dispersed in water, they spontaneously form closed structure with internal aqueous environment bounded by phospholipid bilayer membranes, this vesicular system is called as liposome ${ }^{2}$.Liposomes are the small vesicle of spherical shape that can be produced from cholesterols, non toxic surfactants, sphingolipids, glycolipids, long chain fatty acids and even membrane proteins ${ }^{3}$. Liposomes are the drug carrier loaded with great variety of molecules such as small drug molecules, proteins, nucleotides and even plasmids. Liposome can be formulated and processed to differ in size, composition, charge and lamellarity. To date liposomal formulations of anti-tumor drugs and antifungal agents have been commercialized ${ }^{4}$. The clinical potential of liposomes as a vehicle for replacement therapy in genetic deficiencies of lysosomal enzymes was first demonstrated in 1970s 5, 6. Considerable progress was made during 1970s and 1980s in the field of liposome stability leading to long circulation times of liposomes after intravenous administration resulting in the improvement in bio-distribution of liposome. The important anti-tumour drug doxorubicin had been formulated as liposome in 1980s to improve the therapeutic index. Liposome research is gaining importance in biological, pharmaceutical and medical research because liposomes seem to be the most effective carriers for the introduction of all kinds of agents into cells ${ }^{7}$.The efficacy of liposomes as drug delivery systems would be increased dramatically if it were possible to deliver their contents selectively to particular cells or anatomical sites. Several types of liposomal formulations have been explored; such as those bearing carbohydrate determinants (lectins), monoclonal antibodies, glycoproteins and antigens with a view of targeting the liposomes to selective tissues or anatomical sites ${ }^{\mathbf{8 , 9}}$.However, most of these formulations have failed to show any remarkable therapeutic efficacy because of several reasons such as antigenicity of the ligands, inability of the drug to reach the appropriate cellular compartment in active form and the presence of the endothelial or other histological barrier between the liposomes and its cellular binding site. These difficulties can be minimized by using temperature sensitive liposomes. These liposomes were developed by Yatvin et 
$\mathrm{al}^{10}$ Weinstein et al. and others for better orientation to targeted sites. These liposomes are formed by a mixture of synthetic lipids such as dipalmitoylphosphatidylcholine (DPPC) and distearoylphosphatidylcholine (DSPC), that exhibits a gel to sol transition at temperature few degrees above the physiological temperature, a range easily obtainable by local hyperthermia. It was postulated that such liposomes would remain reasonably stable in the vascular system at the normal body temperature $\left(37^{\circ} \mathrm{C}\right)$, and when passing through the heated area they would release their contents when the transition temperature is attained ${ }^{11,12,13}$.

There are several mechanisms by which liposomes act within and outside the body which are as follows ${ }^{14}$

1- Liposome attaches to cellular membrane and appears to fuse with them, releasing their content into the cell.

2- 2- Some times they are taken up by the cell and their phospholipids are incorporated into the cell membrane by which the drug trapped inside is released

3- 3- In the case of phagocyte cell, the liposomes are taken up, the phospholipid walls are acted upon by organelles called lysosomes and the active pharmaceutical ingredients are released.[lectr11 note ]

\section{ADVANTAGE OF LIPOSOMES}

- $\quad$ Provide controlled drug delivery

- Biodegradable, biocompatible, flexible

- Non ionic

- Can carry both water soluble and lipid soluble drugs

- Drug can be stabilized from oxidation

- Controlled hydration

- Improve protein stabilization

- Provide sustained release

- Targeted drug delivery and site specific drug delivery

- Stabilization of entrapped drug from hospital environment

- Altered pharmacokinetics and pharmacodynamics

- Can be administered through various route

- Act as reservoir of drug

- Therapeutic index of drug is increased

- Site of avoidance therapy

- Can modulated the destitution of drug

- $\quad$ Direct interaction of drug with cell

\section{METHODS OF LIPOSOME FORMATION AND DRUG LOADING}

There are many ways of preparing lipososmes as listed below. Some of the important methods have been explained.

1. Hydration of lipids in presence of solvent

2. Ultrasonication

3. French Pressure cell

4. Solvent injection method

(C) 2011-14, JDDT. All Rights Reserved a) Ether injection method

b) Ethanol injection

5. Detergent removal Detergent can be removed by
a) Dialysis
b) Coloumn chromatography
c) Bio-beads

6. Reverse phase evaporation technique

7. High pressure extrusion

8. Miscellaneous methods

a) Slow swelling in Non electrolyte solution

b) Removal of Chaotropic ion

c) Freeze-Thawing

Convectional method ${ }^{15}$

In the procedure; the phospholipids are dissolved in an organic solvent (usually a chloroform/methanol mixture) and deposited from the solvents as a thin film on the wall of a round bottom flask by use of rotary evaporation under reduced pressure. MLVs form spontaneously when an excess volume of aqueous buffer containing the drug is added to the dried lipid film. Drug containing liposomes can be separated from nonsequestered drug by centrifugation of the liposomes or by gel filtration. The time allowed for hydration of the dried film and conditions of agitation are critical in determining the amount of the aqueous buffer (or drug solution) that will be entrapped within the internal compartments of the MLVs.

\section{Sonication method ${ }^{15}$}

This method is used in the preparation of SUVs and it involves the subsequent sonication of MLVs prepared by the conventional method either with a bath type or a probe type sonicator under an inert atmosphere, usually nitrogen or argon. The principle of sonication involves the use of pulsed, high frequency sound waves (sonic energy) to agitate a suspension of the MLVs. Such disruption of the MLVs produces SUVs with diameter in the range of $15-50 \mathrm{~nm}$. The purpose of sonication, therefore, is to produce a homogenous dispersion of small vesicles with a potential for greater tissue penetration. The commonly used sonicators are of the bath and probe tip type. The major drawbacks in the preparation of liposomes by sonication include oxidation of unsaturated bonds in the fatty acid chains of phospholipids and hydrolysis to lysophospholipids and free fatty acids. Another drawback is the denaturation or inactivation of some thermolabile substances (e.g., DNA, certain proteins, etc) to be entrapped.

\section{High-pressure extrusion method ${ }^{15}$}

This is another method for converting MLV to SUV suspensions. By this method, suspensions of MLVs prepared by the convectional method are repeatedly passed through filters polycarbonate membranes with very small pore diameter $(0.8-1.0 \mu \mathrm{m})$ under high pressure up to 250psi. By choosing filters with appropriate pore sizes, liposomes of desirable diameters can be produced. The mechanism of action of the high pressure extrusion method appears to be much like peeling an onion. As the MLVs are forced through the ISSN: 2250-1177
CODEN (USA): JDDTAO 
small pores, successive layers are peeled off until only one remains. Besides reducing the liposome size, the extrusion method produces liposomes of homogeneous size distributions. A variety of different lipids can be used to form stable liposomes by this method. Extrusion at low pressures <1Mpa is possible when lipid concentration is low, but the most commonly used pressures are about 5Mpa. A new technique uses $10.5 \mathrm{Mpa}$ for better results.

\section{Solubilization and detergent removal method ${ }^{15}$}

This method is used in the preparation of LUVs and it involves the use of detergent (surfactant) for the solubilisation of the lipids. Detergents used include the non-ionic surfactants [e.g., n-octyl-bete-D-glucopyranose (octyl gluside), anionic surfactants (e.g., dodecyl sulphate) and cationic surfactants (e.g., hexadecyltrimethyl ammoniumbromide). The procedure involves the solubilisation of the lipids in an aqueous solution of the detergent and the protein(s) to be encapsulated. The detergent should have a high critical micelle concentration (CMC), so that it is easily removed. The detergent is subsequently removed by dialysis or column chromatography. During detergent removal, LUVs of diameter $0.08-0.2 \mu \mathrm{m}$ are produced. This detergent removal method has been found suitable for the encapsulation of proteins of biomedical importance.

\section{Reverse phase evaporation technique ${ }^{15}$}

It consists of a rapid injection of aqueous solution of the drug into an organic solvent, which contains the lipid dissolved with simultaneous bath sonication of the mixture leading to the formation of water droplets in the organic solvent (i.e., a "water-in-oil" emulsion). The resulting emulsion is dried down to a semi solid gel in a rotary evaporator. The next step is to subject the gel to vigorous mechanical agitation to induce a phase reversal from water-in-oil to oil-inwater dispersion (i.e., an aqueous suspension of the vesicles). During the agitation, some of the water droplets collapse to form the external phase while the remaining portion forms the entrapped aqueous volume. Large unilamellar vesicles (diameter $0.1-1 \mu \mathrm{m}$ ) are formed in the process. This method has been used to encapsulate both small and macromolecules such as RNA and various enzymes without loss of activity. The expected limitation of this method is the exposure of the material to be encapsulated to organic solvents and mechanical agitation, which can lead to the denaturation of some proteins or breakage of DNA strands. Reports of such limitations are however rare in the literatures.

\section{APPLICATIONS OF LIPOSOMES}

\section{Applications of liposomes in medicine}

Applications of liposomes in pharmacology and medicine can be divided into therapeutic and diagnostic applications of liposomes containing drugs or various markers, and their use as a model, tool, or reagent in the basic studies of cell interactions, recognition processes, and of the mode of action of certain substances.Unfortunately many drugs have a very narrow therapeutic window, meaning that the therapeutic

concentration is not much lower than the toxic one. In several cases the toxicity can be reduced or the efficacy enhanced by the use of an appropriate drug carrier which changes the temporal and spatial distribution of the drug, i.e. its pharmacokinetics and biodistribution ${ }^{16}$.

\section{A. Modes of liposome action}

Liposomes as drug delivery systems can offer several advantages over conventional dosage forms especially for parenteral (i.e. local or systemic injection or infusion), topical, and pulmonary route of administration. The preceding discussion shows that liposomes exhibit different biodistribution and pharmacokinetics than free drug molecules. In several cases this can be used to improve the therapeutic efficacy of the encapsulated drug molecules. The limitations can be reduced bioavailability of the drug, saturation of the cells of the immune system with lipids and potentially increased toxicity of some drugs due to their increased interactions with particular cells. The benefits of drug loaden liposomes, which can be applied as (colloidal) solution, aerosol, or in (semi) solid forms, such as creams and gels, can be summarized into seven categories:

(i) Improved solubility of lipophilic and amphiphilic drugs. Examples include Porphyrins, Amphotericin B, Minoxidil, some peptides, and anthracyclines, respectively; furthermore, in some cases hydrophilic drugs, such as anticancer agent Doxorubicin or Acyclovir can be encapsulateded in the liposome interior at concentrations several fold above their aqueous solubility. This is possible due to precipitation of the drug or gel formation inside the liposome with appropriate substances encapsulated ${ }^{17}$;

(ii) Passive targeting to the cells of the immune system, especially cells of the mononuclear phagocytic system (in older literature reticuloendothelial system).

Examples are antimonials, Amphotericin B, porphyrins and also vaccines, immunomodulators or (immuno)supressors;

(iii) Sustained release system of systemically or locally administered liposomes. Examples are doxorubicin, cytosine arabinose, cortisones, biological proteins or peptides such as vasopressin;

(iv) Site-avoidance mechanism: liposomes do not dispose in certain organs, such as heart, kidneys, brain, and nervous system and this reduces cardio-, nephro-, and neuro-toxicity. Typical examples are reduced nephrotoxicity of Amphotericin B, and reduced cardiotoxicity of Doxorubicin liposomes;

(v) Site specific targeting: in certain cases liposomes with surface attached ligands can bind to target cells ('key and lock' mechanism), or can be delivered into the target tissue by local anatomical conditions such as leaky and badly formed blood vessels, their basal lamina, and capillaries. Examples include anticancer, antiinfection and antiinflammatory drugs;

(vi) Improved transfer of hydrophilic, charged molecules such as chelators, antibiotics, plasmids, and genes into cells.

\section{B. Conventional liposomes}


For historical reasons we shall conventional liposomes distinguish between conventional liposomes and liposomes with altered surface properties. The first generation of liposomes includes various lipid compositions which changed the physicochemical properties of liposomes in a variety of different ways, but could not significantly alter their biological properties upon intravenous administration which is the most widely used route in medical applications. Therefore, the optimistic goals of antibody sensitised liposomes (immunoliposomes as 'guided missiles'), which gave often very encouraging results in in vitro studies - which are in general performed in the absence of immunoglobulins, complement components, and macrophages -failed in in vivo applications. The first condition for the immunoliposome concept towork is therefore that they escape the clearance by the mononuclear phagocytic system. This was made possible by the introduction of sterically stabilized liposomes in which the presence of surface grafted hydrophilic polymers substantially prolongs the liposome blood circulation times, probably due to reduced interactions with the components of the immune system. This reduction arises from the presence of a steric barrier which prevents adsorption or hydrophobic binding of immune system components onto the foreign body. The liposomes with altered surfaces therefore include sterically stabilized liposomes and immunoliposomes. With respect to sterically stabilized immunoliposomes one should add a note of caution. Even liposomes with prolonged circulation in blood are not likely to be as widely applicable as many researchers envision(ed). The main limitations are extravasation (escaping from the blood circulation) and poor blood circulation in solid tumours. In the latter case, some of the particulates suspended in blood which come in the area extravasate due to leaky capillaries and stay or get stuck in the region of the extravasation and actually the presence of surface attached homing ligand, i.e. active targeting, does not really have much influence. In addition to some other targeting possibilities, such as injections in different body cavities immunoliposomes present a viable option in immunoassays and diagnostic tests ${ }^{16}$.

\section{i. Liposomes in parasitic diseases and infections}

Since conventional liposomes are digested by phagocytic cells in the body after intravenous administration, they are ideal vehicles for the targetting of drug molecules into these macrophages. The best knownM examples of this 'Trojan horse-like' mechanism are several parasitic diseases which normally reside in the cell of mononuclear phagocytic system. They include leishmaniasis and several fungal infections. $\mathrm{N}$ Leishmaniasis is a parasitic infection of macrophages which affects over 100 million people in tropical regions and is often fatal. The efficacious dose of drugs, mostly different antimonials, is not much lower than the toxic one. Liposomes accumulate in the very same cell population which is infected and therefore offer an ideal drug delivery vehicle ${ }^{16}$. Indeed, the therapeutic index was increased in rodents as much as several hundred times upon administration of the drug in various liposomes. Surprisingly, and unfortunately, there was not much interest to scale up the formulations and clinically approve them after several very encouraging studies dating back to 1978 . Only now, there are several ongoing studies with various antiparasitic liposome formulations in humans. These formulations use mostly ionophore Amphotericin B and are transplanted from very successful and prolific area of liposome formulations in antifungal therapy. The best results reported so far in human therapy are probably liposomes as carriers for Amphotericin B in antifungal therapies. This drug is the drug of choice in disseminated fungal infections which often parallel compromised immune system, chemotherapy, or AIDS and are frequently fatal. Unfortunately, the drug itself is very toxic and its dosage is limited due to its nephro- and neuro-toxicity. These toxicities are normally correlated with the size of the drug molecule or its complex and obviously liposome encapsulation prevents accumulation of drug in these organs and drastically reduces toxicity ${ }^{16}$. In addition, often the fungus resides in the cells of the mononuclear phagocytic system and therefore the encapsulation results in reduced toxicity and passive targetting. These benefits, however, can be associated with any colloidal drug carrier. Indeed, similar improvements in therapy were observed with microemulsions and stable mixed micellar formulations ${ }^{16}$. Furthermore, it seems that many of the early liposomal preparations were in fact liquid crystalline colloidal particles rather then selfclosed multilamellar liposomes. Since the lives of the first terminally ill patients, which did not respond to all the conventional therapies, were saved ${ }^{16}$ many patients were very successfully treated with a variety of Amphotericin B formulations. Similar approaches can be implemented in antibacterial, and antiviral therapy ${ }^{16}$.Most of the antibiotics, however, are orally available and liposome encapsulation can be considered only in the case of very potent and toxic ones which are administered parenterally. The preparation of antibiotics loaded liposomes at reasonably high drug to lipid ratios may not be easy because of the interactions of these molecules with bilayers and high densities of their aqueous solutions which often force liposomes to float as a creamy layer on the top of the tube. Several other routes, such as topical or pulmonary (by inhalation) are being considered also. Liposome encapsulated antivirals such as acyclovir, ribavarin, or azide thymidine (AZT) have also shown reduced toxicity and currently more detailed experiments are being performed with respect to their efficacy.

\section{ii. Macrophage activation and vaccination}

The automatic targetting of liposomes to macrophages can be exploited in several other ways, including the macrophage activation and in vaccination. Some natural toxins induce strong macrophage response which results in macrophage activation. This can be duplicated and improved by the use of liposomes because small molecules with immunogenic properties (haptens) cannot induce immune response without being attached to a larger particle. For instance, liposomes containing muramyl tripeptide, the smallest bacterial cell wall subunit with immunogenic properties cause macrophage activation. Activated macrophages are larger and contain more granulomae and lysosome material. 


\section{iii. Liposomes in anticancer therapy}

Many different liposome formulations of various anticancer agents were shown to be less toxic than the free drug ${ }^{18}$. Anthracyclines are drugs which stop the growth of dividing cells by intercalating into the DNA and therefore kill predominantly quickly dividing cells. These cells are in tumours, but also in gastrointestinal mucosa, hair, and blood cells and therefore this class of drugs is very toxic. The most used and studied is Adriamycin (commercial name for Doxorubicin $\mathrm{HCl}$ ). In addition to the above mentioned acute toxicities its dosage is limited by its cumulative cardiotoxicity. Many different formulations were tried. In most cases the toxicity was reduced about $50 \%$. This includes both, short term and chronic toxicities because liposome encapsulation reduces the distribution of the drug molecules towards those tissues. For the same reason, on the other hand, the efficacy was in many cases compromised due to the reduced bioavailability of the drug, especially if the tumour was not phagocytic, or located in the organs of mononuclear phagocytic system. In some cases, such as systemic lymphoma, the effect of liposome encapsulation showed enhanced efficacy due to the sustained release effect, i.e. longer presence of therapeutic concentrations in the circulation ${ }^{19}$ while in several other cases the sequestration of the drug into tissues of mononuclear phagocytic system actually reduced its efficacy. Applications in man showed in general reduced toxicity, better tolerability of administration with not too encouraging efficacy. Several different formulations are in different phases of clinical studies and show mixed results ${ }^{20}$.

\section{iv. Other applications}

Small liposomes composed of lipids with long and saturated hydrocarbon chains in mixtures with cholesterol were shown to accumulate at the sites of inflammations. Such liposomes were used for diagnostic purposes $^{21}$. They can also deliver anti-inflammatory drugs. Liposomes containing corticosteroids were injected also directly into the sites of inflammations, especially into arthritic joints where they acted as a sustained release system. Additionally, the contamination of healthy tissues with drug molecules was reduced. Liposomes can be used also to deliver drugs into the lung 22 . This is most often done by inhalation of liposome aerosol. This can be used either for the treatment of various lung disorders, infections, asthma, or using lungs as a drug depot for the systemic delivery.One of the possible applications of these aerosols is in the asthma relief in which the dosing frequency can be substantially reduced and single inhalation can last overnight ${ }^{23}$. The natural fate of liposomes to accumulate in liver and spleen was exploited in the treatment of neonatal jaundice in an animal model ${ }^{24}$. The application of free and liposomal metalloporphyrins which inhibit enzyme which breaks down hemoglobin into toxic bilirubin, however, did not result in statistically significant reduction of the enzyme activity. This is probably due to the fact that uptake by liver greatly exceeds the uptake into the spleen in which the degradation takes place. However, when the liver uptake was presaturated with a dose of empty liposomes, the enzymatic activity was significantly reduced due to targetting of liposomes to the spleen ${ }^{24}$. Liposomes can be applied also as a thick cream, gel, or tincture. In addition to subcutaneous or intramuscular drug depot these formulations can be applied topically. Several researchers claim increased penetration of lipid and drug molecules into the skin. These data, as well as possible mechanisms, are, however, still a matter of controversy. Oral applications of liposomes are at present rather limited due to the very liposomicidal environment in stomach and duodenum and normally the administration of free or liposome encapsulated drug exhibits usually no differences. Intragastrical administration, however, shows that liposomes enhance the systemic bioavailability of certain water insoluble drugs and vitamins. Several designs to stabilize liposomes in low $\mathrm{pH}$, degradative enzyme, and bile salts containing environments are being studied. They include liposomes composed from many bilayers with different chemical stability and with programmable degradation kinetics, liposome encapsulated in biodegradable gels or capsules, polymer coated liposomes, and similar. More research is needed, however, to find out if some of these approaches are commercially viable.

\section{Liposomes with altered surface properties}

All these applications have made use of the so-called conventional liposomes. New strategies, including selective targetting of cancer and other diseased cells, however, rely on liposomes with altered surface properties. For selective interactions with particular cells, liposomes have to bear surface attached antigens. The application of these immunoliposomes, however, suffers from their quick clearance from the blood by the immune system and their inability to extravasate, i.e. leave the blood stream. These two limitations can be bypassed in certain applications, such as in treatments of intraperitoneal tumours or other disorders, in the use of liposomes as localized drug reservoir, in some topical applications, or in pulmonary applications of liposome aerosols, but for the majority of other applications the fast clearance represents the major obstacle. For this reason sterically stabilised liposomes were introduced which can largely avoid detection by the immune system and were shown to have blood circulation times for several days (half lives in humans $>2$ days as compared to minutes rather than hours of conventional liposomes). For this reason they are often called Stealth liposomes. Of course only with stealth immunoliposomes, systemic active targetting became a possibility ${ }^{16}$.

\section{* Sterically stabilised liposomes}

The fate of liposomes, i.e. their rapid clearance from the body, was realized rather early. First attempts to alter their biodistribution by either surface ligands or membrane composition were undertaken in the late 70's. The results showed that liposome disposition can be altered, but predominantly within the mononuclear phagocytic system including the intrahepatic uptake itself. Blood circulation times were prolonged but the first substantial improvements were achieved by the incorporation of ganglioside GM1 or phosphatidylinositol at 5-10 $\mathrm{mol} \%$ into the bilayer. The best results were obtained by substituting these two 
lipids with synthetic polymer containing lipids. The longest circulation times were achieved when polyethylene glycol covalently bound to the phospholipid was used. It seems that intermediate molecular weights, from 1500 to $5000 \mathrm{Da}$ are the optimum .The origin of steric stabilisation is well documented although not well understood. It was shown that the Alexander-de-Gennes model of polymers at interfaces can qualitatively explain the stability of liposomes in biological systems. The model can explain minimal polymer concentration above the surface of the bilayer at which polymer forms the so-called brush conformation and which acts as a steric shield. Small angle X-ray scattering measurements of force-distance profiles of polyethylene glycol grafted liposomes have shown enhanced bilayer repulsion in agreement with the hypothesis that reduced surface adhesion and adsorption stabilises liposomes. Recent theoretical work also explained the experimentally well known fact that increased concentrations of longer chains start to reverse the effect at particular polymer density ${ }^{16}$. This is due to the so-called collapse of the brush which occurs at certain polymer density and results in polymer self aggregation, a well known fact from the experimental polymer science. Longer chains can also exhibit increased attractive and bridging forces with macromolecules. Of course, the in vivo and in vitro stability are not necessarily correlated and, for example, in vitro very stable formulations, such as highly charged ones, or the ones with charged brush, are cleared in vivo very rapidly. Another factor which may differ between the two tests is the role of chain flexibility on the interactions with particles and proteins. It is possible that the decreased mobility of chains in the denser brush regimes, when the chain motion correlation times may approach times required for protein binding, can account for the weak physisorption of proteins ${ }^{16}$.

\section{* Medical applications of stealth liposomes}

Sterically stabilised vesicles can act either as long circulating microreservoirs or tumour (or site of inflammation and infection) targetting vehicles. The former applications requires larger liposomes $(\sim 0.2 \mu \mathrm{m})$ while the latter one is due to the ability of small vesicles to leave the blood circulation. The prolonged presence of small particulates in blood results in effective extravasation in regions with porous, damaged, or badly formed blood vessels which often characterize tumours or their vicinity. While normal molecules and macromolecules quickly come to equilibrium large doses of liposomes can accumulate due to their adhesion or immobilization. (In analogy with biocompatible surfaces we can speculate that PEG chains effectively reduce the adsorption of proteins while for the prevention of cell adhesion much longer chains would be required ${ }^{16}$. At present, it is still not known if such long chains can be effectively incorporated into liposomes.) This allows larger doses of liposome loaded drugs to be delivered to malignant tissues. For instance more then $10 \%$ of the injected dose of stealth liposome encapsulated Doxorubicin was found in tumours ${ }^{25}$ as opposed to around $1 \%$ when free drug was administered. Extensive liposome localization in the tumors was observed. Healthy tissue did not accumulate any signal which was due to Doxorubicin fluorescence ${ }^{16}$. Efficacy studies in various mice tumour models, such as implanted solid C26 carcinoma and inoculated mammary carcinoma, have shown dramatic improvements ${ }^{26,30}$. Solid C26 colon tumour is practically Doxorubucin) liposomes, and mixtures of free drug and empty stealth liposomes. Stealth Epirubicin and Doxorubicin liposomes resulted, however, in complete remissions of tumors in the early treatment schedule and substantial reduction of tumour size in the delayed treatment regime ${ }^{27}$.Similar improvement in therapy was observed also in mammary carcinoma ${ }^{28}$. These formulations were substantially more effective not only in curing mice with recent implants from various tumors but also in reducing the incidence of metastases originating from these intra mammary implants. Similarly, several fold increased drug accumulation was observed also in sites of infections which are also characterized by the enhanced vascular permeability. For instance, in mice with infected lungs 10 fold more antibiotic drug accumulated in the infected lung as compared to the noninfected one ${ }^{29}$. Sterically stabilised liposomes may act also as a sustained drug release system either as a long circulating microreservoir or localised drug depot. The first example is provided by improved therapeutic efficacy of cytosine arabinose in the treatment of lymphoma ${ }^{30}$ while the subcutaneous/intramuscular sustained release system was demonstrated by the action of polypeptide vasopressin ${ }^{32}$. Its action was prolonged up to a month as compared to few days for a free drug and a week for the peptide encapsulated in conventional liposomes. It is important to note that these concepts are becoming more and more important with the introduction of genetically engineered polypeptides and proteins which are hampered by the rapid blood clearance, degradation and/or deactivation in the body. The altered biodistribution of stealth liposomes, in addition to the accumulation at the sites characterised with porous blood capillaries, such as in tumours, inflammations, and infections, may benefit several other applications. In the intact vasculature the distribution of stealth liposomes is shifted from the liver, spleen, and bone marrow towards skin. This opens the opportunity to deliver antivirals and dermatological agents to these sites. On the other hand, and while it was shown that the administration of empty stealth liposomes is well tolerated ${ }^{16}$,it requires careful toxicology and tolerability studies when liposomes loaden with potent drugs are used.

\section{* Applications of Stealth liposomes in man}

The encouraging results of Doxorubicin encapsulated in Stealth liposomes in preclinical studies were observed also in clinical trials in humans. Blood circulation times around 45 hours were found ${ }^{33}$ and at reduced toxicity very good response in AIDS patients with Kaposi sarcoma was observed $^{34,35}$. Long circulation times significantly, i.e. 200-fold, increased the area under curve of drug concentration vs time and accumulation in various tumours was proportional to the liposome circulation times ${ }^{34}$. The drug remained encapsulated in circulating liposomes up to one week after injection while at tumour sites drug metabolites were found indicating that it had been released from liposomes. The concentration of the drug in tumours was 4-10 times 
greater than in control group which was treated with free $\operatorname{drug}^{33}$. The same selective targeting was observed also in patients with Kaposi sarcoma. Practically all patients showed considerable decrease in modularity of skin lesions while total flattening was observed in $25 \%$ of the cases $^{34}$. The high efficacy was due to the approximately ten fold higher drug concentration in lesions as compared to the administration of free drug ${ }^{35}$. In conclusion, it seems that stealth liposomes loaded with anticancer drugs will achieve substantial improvements in the treatments of various tumours. In addition, it is hoped that they will be effective also in the treatments of inflammations, infections, and in antiviral therapy.

\section{Liposomes in bioengineering}

Modern genetic engineering and gene recombinant technology is based on the delivery of genetic material, i.e. fragments of DNA, into various cells and microorganisms in order to alter their genetic code and force them to produce particular proteins or polypeptides $^{36}$. Nucleic acids used in gene transfer are large, with molecular weights up to several million Daltons, highly charged and hydrophilic and therefore not easy to transfer across cell membranes. Additionally to classical methods, such as direct injection, phosphate precipitation and others, liposomes were tried as transfection vectors. They can deliver the encapsulated or bound nucleic acid into cells predominantly in two ways: the classical approach is to encapsulate the genetic material into liposomes and liposomes act as an endocytosis enhancer while recently the phosphate or DEAE precipitation was simulated by liposomes. In these cases the nucleic acid forms a complex with several cationic liposomes and the size of the complex and its adsorption on the cell surface catalyses endocytosis or, possibly, fusion. The third, still unexplored way would be to use fusogenic liposomes or cause fusion upon adsorption of the liposome on the cell surface. The classical approach used predominantly large unilamellar vesicles made from negatively charged phosphatidylserine in order to prevent interaction with DNA molecules which may contain up to several thousand negative charges ${ }^{37}$. In some cases transfection efficiencies were improved several hundred times and plant protoplast which are very difficult to transfect were successfully genetically altered ${ }^{38}$. In the mid 80 's, however, electroporation showed better results and the interest for liposomes markedly diminished. Recently, however, transfection was successfully performed using small unilamellar vesicles made from positively charged lipids. First studies used cationic lipid dioleoyl-propyltrimethylammonium (DOTMA)[57]. Later studies showed better transfection efficiencies by using some of the commercially available cationic lipids ${ }^{40}$. Better transfection efficiencies at reduced toxicity were found by using liposomes containing positively charged cholesterol ${ }^{41}$. Many novel cationic lipids are being synthetised in order to improve transfection, especially in vivo. These methods can be used also in gene therapy. The idea is to deliver the nondefective gene into the appropriate cells and hope that they will respond. For instance, patients with cystic fibrosis have a defective gene that encodes the code for a protein critical to a transfer of salts through the cell membrane in the lungs.
In recent experiments, upon inhalation of the copies of human gene mixed with liposomes, $70 \%$ of the cells lining the lungs of mice incorporated the gene and began using it to make proteins in large amounts ${ }^{42}$. This promises elimination or drastic reduction of the symptoms of the disease if it can be repeated in humans.

\section{Application of Liposomes in cosmetics}

In general the rules for topical drug applications and delivery of other compounds are less stringent than the ones for parenteral administration and several hundred cosmetic products are commercially available since Capture (C. Dior) and Niosomes (L'Or'eal) were introduced in 1987. They range from simple liposome pastes which are used as a replacement for creams, gels, and ointments for do-it-yourself cosmetical products to formulations containing various extracts, moisturizers, antibiotics, and to complex products containing recombinant proteins for wound or sunburn healing. Most of the products are anti-ageing skin creams. Unrinsable sunscreens, long lasting perfumes, hair conditioners, aftershaves and similar products, are also gaining large fractions of the market. Liposomal skin creams already share more than $10 \%$ of the over $\$ 10$ billion market. As in the case of topical delivery in medical applications, the workers in the field do not agree on the mechanism of action. While some claim enhanced permeability into the skin the others claim mostly that liposomes are a noninteractive, skinnonirritating, water based matrix (without alcohols, detergents, oils and other non-natural solubilizers) for the active ingredients ${ }^{16}$.

\section{Application of liposomes in agro-food industry}

The ability of liposomes to solubilize compounds with demanding solubility properties, sequester compounds from potentially harmful milieu, and release incorporated molecules in a sustained and predictable fashion can be used also in the food processing industry. Lipid molecules, from fats to polar lipids, are one of the fundamental ingredients in almost any food. For instance, lecithin and some other polar lipids are routinely extracted from nutrients, such as egg yolks or soya beans. Traditionally polar lipids were used to stabilize water-in-oil and oil-in-water emulsions and creams, or to improve dispersal of various instant powders in water. With the advent of microencapsulation technology, however, liposomes have become an attractive system because they are composed entirely from food acceptable compounds. The sustained release system concept can be used in various fermentation processes in which the encapsulated enzymes can greatly shorten fermentation times and improve the quality of the product. This is due to improved spatial and temporal release of the ingredient(s) as well as to their protection in particular phases of the process against chemical degradation. A classical example is cheese making. The first serious attempts to decrease the fermentation time using cell-wall-free bacterial extracts were encouraging enough to stimulate efforts to improve enzyme presentation. After preliminary studies in which liposome systems were optimized the cheese ripening times can be shortened by $30-50 \%{ }^{43,45}$. This means a substantial economic profit knowing that ripening times 
of some cheeses, such as Cheddar, say, are about one year during which they require well controlled conditions. In addition, due to the better dispersal of the enzymes the texture of cheeses was even and bitterness and inconsistent flavour due to the proteolysis of enzymes in the early phase of fermentation was much improved $^{43,44}$. In addition to improved fermentation, liposomes are being tried in the preservation of cheeses. Addition of nitrates to cheese milk to suppress the growth of spore-forming bacteria is now being questioned due to health concerns and natural alternatives are under study. Lysozyme is effective but quickly inactivated due to binding to cassein. Liposome encapsulation can both preserve potency and increase effectiveness because liposomes become localized in the water spaces between the casein matrix and fat globules of curd and cheese. This also happens to be where most of the spoilage organisms are located ${ }^{45}$. These applications of enhancing natural preservatives, including antioxidants such as vitamins $\mathrm{E}$ and $\mathrm{C}$, will undoubtedly become very important due to recent dietary trends which tend to reduce the addition of artificial preservatives and ever larger portion of unsaturated fats in the diet. In other areas of the agro-food industry, liposomes encapsulated biocides have shown superior action due to prolonged presence of the fungicides, herbicides or pesticides at reduced damage to other life forms ${ }^{46}$. Liposome surface can be made sticky so that they remain on the leafs for longer times and they do not wash into the ground. In these applications inexpensive liposomes produced from synthetic lipids are used. The same liposomes are being tried in shellfish farms. These animals are susceptible for many parasitic infections. They are filtermfeeders and they pump large amounts of water through their body. This seems to offset large dilutions of liposomes in the pool and the drug molecules as well as some essential nutrients needed in ppm to ppb quantities can be delivered.

\section{REFERENCES}

1. B. Tiwari Sandip, N. Udupa, B. S. S. Rao*, P. Uma Devi*Thermosensitive Liposomes And Localised Hyperthermia - An Effective Bimodality Approach For Tumour Management Indian Journal of Pharmacology 2000; 32: 214-220.

2. Grubber, S.M. Liposome Biophysics Therapeutics, Marcel Dekker: New York, 1987.

3. Bangham, A.D.; Standish, M.M.; Watkens, J.C. J. Mol. Biol., 1965, 13, 238.

4. Jayakrishnan, A.; Latha, M.S. Controlled and Novel Drug Delivery,B.S. Pub: New Delhi, 1997.

5. Sessa, G.; Weismann, G. J. Biol. Chem., 1970, 245, 3295.

6. Gregoriadis, G. Methods Enzymol., 1976, 44, 278.

7. Abdus Samad, Y. Sultana* and M. Aqil Liposomal Drug Delivery Systems: An Update Review Current Drug Delivery, 2007, 4, 297-305.

8. Juliano RL, Layton D. Liposomes as drug delivery system. In: Juliano RL (Ed.) Drug Delivery System. Oxford University Press, Oxford, UK, 1980, 189- 236

9. Huang A, Huang L, Knnel SJ. Monoclonal antibody covalently coupled with fatty acid. J Biol Chem 1980; 255:8015-8.

10. Yatvin MB, Weinstein JN, Dennis WH, Bluementhal R. Desig of liposomes for enhanced local release of drugs by hyperthermia. Science 1978;202:1290-3.

11. Weinstein JN, Magin RL, Cysyk RL, Zaharko DS. Treatment of solid L1210 murine tumors with local hyperthermia and

\section{Other applications of liposomes}

The potential of making large quantities of inexpensive and stable liposomes may put forward several other applications. They range from water based paints, single tube two component glues or resins, self healing paints, and similar products. They are based mostly on the dissolving potential of liposomes and their ability to protect the encapsulated substance until an external stimulus such as the presence of oxygen, light, or change in hydration. Critical evaluation of these applications is difficult, however, because the information is mostly concentrated in progress reports, business analyses, or prospectuses of various producers. In ecology, liposomes offer improvements in bioreclamation and various monitoring and analytical-diagnostic applications. For instance, it was shown that in an oil spill, the addition of various bacteria with possible nutrients encapsulated in liposomes improves the degradation rates of carbohydrates, which are otherwise very slow. Due to the surfactant action liposomes also improve the coagulation and sinking of oil spread on the water surface or its cleaning up with floating booms. The Environmental Protection Agency is testing liposomes' ability to deliver nutrients to oil spills to speed up the degradation. Liposomes containing membrane anchored chelators can be used to clean toxic or radioactive metals from solutions. For instance, water contaminated in a nuclear reactor can be purified by addition of such liposomes which could be easily precipitated after binding of the toxic ions. In addition to the above mentioned liposome applications there are many others which were not mentioned. Despite over a 1 billion dollar cosmetic liposome industry, I dare to say that the real future of liposomes is in anticancer and possibly other chemotherapies, gene therapy as well as some other medical applications such as artificial blood ${ }^{16}$.

temperature-sensitive liposomes containing methotrexate methotrexate. Cancer Res 1980;40:1388-95.

12. Tacker JR, Anderson RU. Delivery of antitumor drug to bladder cancer by use of phase transition liposomes and hyperthermia. J Urology 1982;127:1211-4.

13. Zou YY, Ueno M, Yamagishi M, Horokoshi I, Yamashita I, Tazawa K, Gu XO. Targeting behaviour of hepatic artery injected temperature-sensitive liposomal adriamycin on tumor-bearing rats. Sel Cancer Ther 1990;6:119-27.

14. Dunnick, J. K.; Rooke, J D.; Aragon, S.; Kriss, J. P. Cancer. Res., 1976, 36, 2385-2389

15. Mohanty Sivasankar* and T. Katyayani liposomes - the Mfuture of formulations ,International journal of research in pharmacy and chemistry ISSN: 2231,2781 IJRPC 2011, 1(2)

16. R. Lipowsky and E. Sackmann Applications of Liposomes Handbook of Biological Physics 1995 Elsevier Science B.V. page number 491-517.

17. Lasic, D.D., P.M. Frederik, M.C.A. Stuart, Y. Barenholz and T.J. McIntosh, 1992, Gelation of liposome interior. A novel method for drug encapsulation, FEBS Lett. 312, 255-258.

18. Gabizon, A., 1989, Liposomes as a drug delivery system in cancer therapy, in: Drug Carrier Systems, eds F.H.D. Roerdink and A.M. Kron (Wiley, Chichester) pp. 185-211

19. . Storm, G., F.H. Roerdink, P.A. Steerenberg, W.H. de Jong and D.J.A. Crommelin, 1987, Influence of lipid composition on the antitumor activity exerted by doxorubicin containing 
liposomes in a rat solid tumor model, Cancer Res. 47, 33663372.

20. Lopez-Berestein, G. and I.J. Fidler (eds), 1989, Liposomes in the Treatment of Infectious Diseases and Cancer (A. Liss, New York).

21. Williams, B.D., M.M. Sullivan, K.E. Williams, J.R. Williams and J.R. Morgan, 1986, Imaging in rheumatoid arthritis using liposomes labeled with technetium, Br. Med. J. 293, 1144 1145.

22. McCalden, T., 1990, Particulate systems for drug delivery to the lung, Adv. Drug Del. Rev. 5, 253-263.

23. Fielding, R.M. and R.M. Abra, 1992, Factors affecting the release rate of terbutaline from liposome formulations after intratracheal instillation in the guinea pig model, Pharm. Res. 9, 220-222.

24. . Hamori, C.J., D.D. Lasic, H.J. Vreman and D.K. Stevenson, 1993, Targeting zinc protoporphyrin liposomes to the spleen using reticuloendothelial blockade with blank liposomes, Pediatric Research, 34, 1.

25. Gabizon, A., 1992, Selective tumor localization and improved therapeutic efficacy of anthracyclines encapsulated in long circulating liposomes, Cancer Res. 52, 891-896. 518 D.D. Lasic

26. Gabizon, A., 1992, Selective tumor localization and improved therapeutic efficacy of anthracyclines encapsulated in long circulating liposomes, Cancer Res. 52, 891-896. 518 D.D. Lasic

27. Mayhew, E.G., D.D. Lasic, S. Babbar and F.J. Martin, 1992, Pharmacokinetics and antitumor activity of epirubicin encapsulated in long circulating liposomes incorporating a polyethylene glycol derivatized phospholipid, Int. J. Cancer 51, 302-309

28. J. Vaage, E. Mayhew, D.D. Lasic and F.J. Martin, 1992, Therapy of primary and metastatic mouse mammary carcinoma with doxorubicin encapsulated in long circulating liposomes, Int. J. Cancer, 51, 942-948.

29. Huang, S.K., E. Mayhew, S. Gilani, D.D. Lasic, F.J. Martin and D. Papahadjopoulos, 1992, Pharmacokinetics and therapeutics of sterically stabilized liposomes in mice bearing C-26 colon carcinoma, Cancer Res. 52, 6774-6781.

30. Allen, T.M., T. Mehra, C. Hansen and Y. Chin, 1992, Stealth liposome: An improved sustained release system for arabinofuranosylcytosine, Cancer. Res. 52, 2431-2439.

31. . Bakker-Woudenberg, I., A.F. Lokersee, M. ten Kate and G. Storm, 1992, Enhanced localization of liposomes with prolonged blood circulation times in infected lung tissue, Biochim. Biophys. Acta, 1138, 318.

32. Woodle, M.C., G. Storm, M.S. Newman, J.J. Jekott, L.R. Collins, F.J. Martin and F.C. Szoka, 1992, Prolonged systemic delivery of peptide drugs by long circulating liposomes, Pharm. Res. 9, 260-265.

33. Martin, F., A. Gabizon and F.J. Martin (Eds), 1992, Human pharmacokinetics of doxil (stealth liposome encapsulated doxorubicin), in: UCLA Symposium: Prevention and Treatment of AIDS, Book of Abstracts, Keystone, CO.

34. Bogner J.R., F.D. Goebel, 1995, Phase II open-label trial of doxil (stealth liposomal doxorubicin) in advanced AIDS-KS, in: VIII International Conference on AIDS, in ref. 51, in press. Boca Raton.

35. Northfelt, D.W., L. Kaplan, J. Russell, P.A. Volberding, F.J. Martin, Single dose pharmacokinetics, safety and tumor localization study of doxil in AIDS patients with Kaposi's sarcoma, in: VIII International Conference on AIDS, in ref. 51 , in press.

36. Nicolau, C. and A. Cudd, 1989, Liposomes as carriers of DNA, Crit. Rev. Therap. Drug Carr. Systems 6, 239-271.

37. Fraley, R.T, S. Subramani, P. Berg and D. Papahadjopoulos, 1980, Introduction of liposome encapsulated SV40DNA into cells, J. Biol. Chem. 255, 10431-10435.

38. Lurquin, P., 1979, Entrapment of plasmid DNA by liposomes and their interaction with plant protoplasts, Nucl. Acids Res. 6, 3773-3777.

39. Felgner, P., T.R. Gadek, M. Holm, R. Roman, M.Wenz, J.P. Northrop, G. Ringold andM. Danielsen, 1987, Lipofectin: A highly efficient, lipid mediated DNA transfection procedure, Proc. Nat. Acad. Sci. USA 84, 7413-7417.

40. Rose, J.K., L. Buoncore and M.A. Whitt, 1991, A new cationic liposome reagent mediating nearly quantitative transfection of animal cells, Biotechniques 10, 520-525.

41. Gao, X. and L. Huang, 1991, A novel cationic liposome reagent for efficient transfection of mammalian cells, Biophys. Biochem. Res. Commun. 179, 280-285.

42. Stribling, R., E. Brunette, D. Liggett, K. Gaenslar and R. Debs, 1992, Aerosol gene delivery in vivo, Proc. Nat. Acad. Sci. USA 89, 11277-11281.

43. Law, B.A., J.S. King, 1991, Use of liposomes for proteinase addition to cheddar cheese, J. Diary Res. 52, 183-188.

44. Alkhalaf, W., J.C. Piard, M. el Soda, J.C. Gripon, M. Desmezeaud and L. Vassal, 1988, Liposomes as proteinases carriers for the accelerated ripening of St. Paulin type cheese, J. Food Sci. 53, 1674-1679.

45. Kirby, C., 1990, Delivery systems for enzymes, Chem. Br., Sept. 1990, 847-851.

46. Tahibi, A., J.D. Sakurai, R. Mathur and D.F.H. Wallach, 1991, Novasome vesicles in extended pesticide formulation, Proc. Symp. Contr. Rel. Bioact. Mat. 18, 231-232. 